\title{
Antifungal Drug Therapeutic Monitoring: What are the Issues?
}

\author{
Eric Myers ${ }^{1}$ - Elizabeth Dodds Ashley ${ }^{2}$
}

Published online: 8 April 2015

(C) Springer International Publishing AG 2015

\begin{abstract}
Therapeutic drug monitoring (TDM), an established practice for many antimicrobials, has recently seen increasing utilization in the management of patients receiving antifungal agents. There is a growing body of literature supporting the use of TDM for itraconazole, voriconazole, posaconazole, and flucytosine. In addition, clinical practice guidelines have been recently published that give recommendations on the appropriate use of TDM for antifungal agents. However, there are still uncertainties regarding the optimal use of antifungal TDM in clinical practice. We conducted a review of recent literature in order to describe the clinical situations and specific antifungal agents for which TDM is ideal and summarize key information about the pharmacokinetics, pharmacodynamics, drug toxicities, TDM concentration targets, and dose adjustment algorithms for antifungals in which routine TDM is performed.
\end{abstract}

Keywords Therapeutic drug monitoring · Antifungal · Itraconazole · Voriconazole $\cdot$ Posaconazole $\cdot$ Isavuconazole . Flucytosine

This article is part of the Topical Collection on Mycology

Elizabeth Dodds Ashley

Elizabeth_Doddsashley@URMC.Rochester.edu

Eric Myers

Eric_Myers@URMC.Rochester.edu

1 Strong Memorial Hospital, 601 Elmwood Avenue Box 638, Rochester, NY 14642, USA

2 Strong Memorial Hospital, 601 Elmwood Avenue, Rochester, NY 14642, USA

\section{Introduction}

The last two decades have brought significant advances in treatment for invasive fungal disease. These include the availability of multiple oral agents demonstrating broad spectrums of activity with proven efficacy in treating and preventing invasive fungal diseases [1]. This has greatly advanced care for patients with these infections, however has also introduced new uncertainties related to drug exposure. Therapeutic drug monitoring (TDM) is an established practice for many drugs today such as vancomycin, aminoglycosides, antiepileptics, and immunosuppressants. Agents that are ideal candidates for routine TDM demonstrate three key characteristics - a high degree of inter-patient variability in dose-exposure relationship, an established relationship between drug exposure and either efficacy, safety, or both, and an assay that is able to accurately measure drug concentrations. Increased understanding of antifungal pharmacokinetics and pharmacodynamics has led to a growing role for TDM of certain antifungals in routine clinical practice. Currently, there is evidence to support the use of TDM for itraconazole, voriconazole, posaconazole, and flucytosine. A number of review articles have been published that describe the rationale and benefit of TDM for certain antifungal agents [2-5]. More recently, clinical guidelines have been published by the British Society for Medical Mycology (BSMM), as well as the Japanese Society of Chemotherapy and the Japanese Society of Therapeutic Drug Monitoring (JSC/JSTDM) [1, 6]. The purpose of this article is to describe the antifungal agents for which we conduct TDM, and outline its role based on a review of recent literature.

\section{Situations That Favor TDM of Antifungals}

There are numerous clinical circumstances that can increase the variability in dose-exposure relationship in antifungal 
agents. These include patients that display altered or unpredictable pharmacokinetics such as critically ill patients, patients with severe renal or hepatic dysfunction, extremes of age, and obesity [2]. Secondly, given that the azole antifungals are extensively metabolized via the cytochrome p450 enzymes, there are numerous drug-drug interactions that can alter serum concentrations of these medications [7]. Poor oral bioavailability is another factor that plays a large role in the high degree of variability in serum concentrations of certain antifungals, most notably itraconazole and posaconazole. Patients with conditions that impact oral absorption, such as diarrhea or mucositis, are therefore also good candidates for TDM. Severity of infection should also be kept in mind, as TDM is more likely to be useful in treatment of severe disseminated disease or prophylaxis in neutropenic patients at high risk for life-threatening fungal infections. Another situation in which TDM of antifungals has been advocated for is in the assessment of compliance, although less costly alternatives, such as medication refill histories, may be preferable if available.

Perhaps the most important question to ask prior to conducting TDM is whether or not the resulting concentration will alter the treatment plan. For example, if a patient with a confirmed invasive fungal infection is clinically failing therapy on an antifungal agent, it would generally be in the patient's best interest to switch to an alternative agent as opposed to waiting for a concentration to come back prior to making a decision; particularly when the long turn-around time is considered. Conversely, neutropenic cancer patients receiving posaconazole suspension as prophylaxis against invasive fungal infection represent very good candidates for TDM. Since patients are not yet infected, posaconazole concentration is the only available indication of whether or not they have adequate exposures. In addition, these patients often have poor oral intake and other conditions that predispose them to variable absorption.

\section{Assay Availability}

There are two types of assay that have been used for measuring antifungal drug concentrations-microbiological assays or bioassays, and chromatographic assays including liquid chromatography-mass spectrometry (LC-MS) and highperformance liquid chromatography (HPLC). Microbiological assays are relatively inexpensive; however, their major drawback is reduced accuracy due to the potential for interference with other drugs, particularly in the setting of combination antifungal therapy [8].

Overall, concentrations obtained with chromatographic assays and bioassays are similar for patients receiving a single antifungal agent [2]. The exception to this is itraconazole where the production of an active metabolite, hydroxy- itraconazole, cannot be differentiated by bioassay. Caution must be used when converting between itraconazole concentrations measured by bioassay and those measured by HPLC, as the cited degree to which bioassay overestimates HPLCmeasured concentration varies in the literature, with a range of a 2-7-fold overestimation being reported depending on the source $[1,2,9]$. This may not have significant clinical impact however, as it is a common clinical practice to combine itraconazole and hydroxy-itraconazole concentrations when describing itraconazole exposure.

Given these considerations, chromatographic assays are the preferred and most commonly utilized assays for antifungal TDM. Chromatographic assays demonstrate high sensitivity and specificity $[10,11]$. Unfortunately, these assays are only performed by a relatively small number of specialized laboratories, and as a result, it can take a week or more to receive results. Turn-around time is therefore an important consideration when designing an optimal TDM approach for a specific institution.

\section{Antifungals Without a Current Role for TDM}

A clear exposure-response relationship has been documented for both amphotericin B and the echinocandins [12]. However, current pharmacokinetic data does not suggest that there is enough variability in dose-exposure relationship to warrant routine TDM for patients on these therapies [4]. As both classes are only available in IV formulations, there are no issues with variable absorption. There is potential for an expanded role for TDM of glucan synthesis inhibitors in the future, as there is currently an oral enfumafungin derivative, a class of glucan synthesis inhibitors structurally distinct from the echinocandins, undergoing a phase 2 trial for treatment of invasive candidiasis, but more data are needed [13].

Fluconazole demonstrates a well-documented exposure-response relationship, with an AUC/MIC $>50$ associated with clinical efficacy [14-16]. However, due to its excellent oral bioavailability and linear kinetics, it lacks the dose-exposure variability required to consider TDM [17]. Certain populations, such as clinically unstable patients or patients receiving renal replacement therapy, can still experience unpredictable dose-exposure relationships which could make TDM more appropriate. Illustrating this, van der Elst et al. demonstrated a wide range of fluconazole serum concentrations (2.6$46.5 \mathrm{mg} / \mathrm{L}), 40 \%$ of which were subtherapeutic, in critically ill pediatric cancer patients [18]. However, even among these special populations, the lack of a clear target limits the role of TDM for fluconazole as all of the pharmacodynamic data establishing exposure-efficacy relationship utilizes AUC/ MIC, which is not a clinically practical monitoring parameter. The remainder of this review will focus on agents with a demonstrated role for TDM. 


\section{Itraconazole}

\section{Inter-Patient Variability}

Extensive dose-exposure variability of itraconazole has been reported in pharmacokinetic analyses, with the most important contributing factor being oral absorption [19]. The effect of gastric $\mathrm{pH}$ and food on absorption of itraconazole varies based on the formulation used - the capsules require an acidic $\mathrm{pH}$ and administration with food for optimal absorption, while the solution is absorbed best on an empty stomach [20]. Overall, the solution results in better exposure than the capsules; however, patients on either formulation still experience a high degree of variability [19]. Other factors that can alter oral absorption of itraconazole include mucositis, diarrhea, and acid suppressants. Itraconazole is primarily metabolized by CYP3A4, and inducers or inhibitors of this enzyme can also affect drug concentrations [2].

\section{Concentration-Efficacy Relationship}

There are a number of small studies that demonstrate a relationship between itraconazole trough concentration and efficacy for both prophylaxis of invasive fungal infection in immunocompromised patients as well as treatment of invasive fungal infection (Table 1). Studies examining the use of itraconazole for prophylaxis of invasive fungal infections in neutropenic patients have associated concentrations of 0.25 $0.5 \mathrm{mg} / \mathrm{L}$ with efficacy [21-23]. Concentration breakpoints established as potential targets for successful treatment of invasive fungal infection range from 5 to $6.5 \mathrm{mg} / \mathrm{L}$ when measured by bioassay and $0.6-1 \mathrm{mg} / \mathrm{L}$ when measured by HPLC [19, 24-28]. There are a number of limitations associated with interpreting results of these studies, as they are retrospective, include a small number of patients, do not have standardized reasons or timing for obtaining concentrations, and are not designed to establish an ideal concentration target.

Most clinical practice guidelines recommend that trough concentrations be maintained over $0.5 \mathrm{mg} / \mathrm{L}$ for prophylaxis and at least $1 \mathrm{mg} / \mathrm{L}$ for treatment, measured by HPLC (Table 2). The BSMM guidelines recommend a minimum trough concentration of $0.5 \mathrm{mg} / \mathrm{L}$ for both prophylaxis and treatment, although based on the limited evidence available, there seems to be more support for a treatment trough target of at least $1 \mathrm{mg} / \mathrm{L}$ [1]. It is also important to consider that ideal concentrations likely vary based on the MIC of the infecting organism and site of infection, but there is insufficient data to support more tailored targets at this point.

\section{Concentration-Toxicity Relationship}

Adverse events attributable to itraconazole have been thoroughly described in other antifungal pharmacology reviews and include nausea and diarrhea, liver function abnormalities, a negative inotropic effect, and mineralocorticoid effects leading to edema, hypokalemia, and hypertension [20,56]. A retrospective chart review by Lestner et al. demonstrated a clear positive correlation between serum concentration and risk of adverse events, and the authors proposed a concentration of $17.1 \mathrm{mg} / \mathrm{L}$ measured by bioassay as an upper limit for preventing toxicity. Based on CART analysis, $86 \%$ of patients at or above this concentration would be predicted to experience toxicity versus only $31 \%$ of patients below this concentration [57].

In line with these results, the BSMM guidelines recommend that $17 \mathrm{mg} / \mathrm{L}$ (measured by bioassay) is a reasonable limit in order to reduce risk of toxicity [1]. Other clinical guidelines and review articles on antifungal TDM do not take a strong stance on upper limit itraconazole concentrations to reduce toxicity (Table 2).

\section{Recommendations}

Both Andes et al. and the BSMM guidelines recommend routine monitoring of itraconazole concentrations after the initiation of therapy or dose changes independent of indication [1, 2]. Due to the highly variable oral absorption of itraconazole, TDM is appropriate for most patients receiving the drug. Because of the long half-life of itraconazole, it can often take a week for the drug to reach steady state conditions, and the first measurement should not be taken before this point. [20] Concentrations should ideally be measured as trough concentrations; however, due to the long half-life of itraconazole, concentrations drawn in the middle of the dosing interval do not differ substantially from troughs.

After a patient reaches steady state therapeutic concentrations, recommendations on when to check serum concentrations are less defined, but rechecking can be considered for patients who are initiating or discontinuing major interacting medications, who have new concerns about oral absorption, or who demonstrate lack of response or signs of toxicity $[1,2]$. As previously mentioned, concentrations should only be monitored if the results are likely to impact clinical decisionmaking while keeping in mind that the evidence supporting specific targets is limited.

\section{Dose Adjustments}

Dose adjustment algorithms for patients with sub- or supra-therapeutic concentrations of other azole agents have been published recently; however, recommendations for itraconazole are sparse. The BSMM guidelines recommend that the dose be increased from $200 \mathrm{mg}$ twice daily to $300 \mathrm{mg}$ twice daily for patients with subtherapeutic concentrations, but no citation is given [1]. Initial interventions for patients with subtherapeutic itraconazole concentrations 
Table 1 Literature summary of concentration targets associated with clinical efficacy

\begin{tabular}{|c|c|c|c|c|c|}
\hline & Population/indication & $n$ & Concentration & Assay & Reference \\
\hline \multicolumn{6}{|c|}{ Itraconazole } \\
\hline \multirow[t]{3}{*}{ Ppx } & IFI Ppx, hematologic malignancy & 72 & $0.25 \mathrm{mg} / \mathrm{L}$ & HPLC & {$[21]$} \\
\hline & IFI Ppx, hematologic malignancy & 42 & $0.25 \mathrm{mg} / \mathrm{L}$ & HPLC & [22] \\
\hline & IFI Ppx, hematologic malignancy & 20 & $0.5 \mathrm{mg} / \mathrm{L}$ & HPLC & {$[23]$} \\
\hline \multirow[t]{6}{*}{ Tx } & Treatment of severe IFI & 8 & $5 \mathrm{mg} / \mathrm{L}$ & Bioassay & {$[24]$} \\
\hline & Invasive aspergillosis & 21 & $6.5 \mathrm{mg} / \mathrm{L}$ & Bioassay & {$[25]$} \\
\hline & Nonmeningeal coccidioidomycosis & 39 & $6.5 \mathrm{mg} / \mathrm{L}$ & Bioassay & {$[26]$} \\
\hline & Candida denture stomatitis & 36 & $0.6 \mathrm{mg} / \mathrm{L}$ & HPLC & {$[27]$} \\
\hline & Cryptococcal meningitis & 25 & $1 \mathrm{mg} / \mathrm{L}$ & HPLC & {$[28]$} \\
\hline & Candidiasis in AIDS patients & 31 & $1 \mathrm{mg} / \mathrm{L}$ & HPLC & [19] \\
\hline \multicolumn{6}{|c|}{ Voriconazole } \\
\hline \multirow[t]{2}{*}{ Ppx } & IFI Ppx, lung transplant & 93 & $1.5 \mathrm{mg} / \mathrm{L}$ & HPLC & [29] \\
\hline & IFI Ppx, HSCT & 71 & $2 \mathrm{mg} / \mathrm{L}$ & HPLC & {$[30]$} \\
\hline \multirow[t]{11}{*}{ Tx } & Invasive aspergillosis & 122 & $0.25 \mathrm{mg} / \mathrm{L}$ & HPLC & {$[31]$} \\
\hline & Treatment of IFI & 14 & $1 \mathrm{mg} / \mathrm{L}$ & HPLC & {$[32]$} \\
\hline & Treatment of IFI & 108 & $1 \mathrm{mg} / \mathrm{L}$ & HPLC & [33] \\
\hline & Treatment of IFI (peds) & 30 & $1 \mathrm{mg} / \mathrm{L}$ & HPLC & {$[34]$} \\
\hline & Treatment of IFI & 52 & $1 \mathrm{mg} / \mathrm{L}$ & HPLC & [35] \\
\hline & Treatment of IFI & 163 & $1.7 \mathrm{mg} / \mathrm{L}$ & HPLC & {$[36]$} \\
\hline & Treatment of IFI & 34 & $2 \mathrm{mg} / \mathrm{L}$ & HPLC & [37] \\
\hline & Treatment of IFI & 23 & $2 \mathrm{mg} / \mathrm{L}$ & HPLC & [38] \\
\hline & Treatment of IFI & 28 & $2.05 \mathrm{mg} / \mathrm{L}$ & HPLC & [39] \\
\hline & Treatment of IFI & 25 & $2.2 \mathrm{mg} / \mathrm{L}$ & HPLC & {$[40]$} \\
\hline & Treatment or Ppx of IFI & 246 & No correlation & HPLC & {$[41]$} \\
\hline \multicolumn{6}{|c|}{ Posaconazole } \\
\hline \multirow[t]{8}{*}{ Ppx } & IFI Ppx, hematologic malignancy & 63 & $0.15 \mathrm{mg} / \mathrm{L}$ & HPLC & {$[42]$} \\
\hline & IFI Ppx, hematologic malignancy & 36 & $0.35 \mathrm{mg} / \mathrm{L}$ & HPLC & {$[43]$} \\
\hline & IFI Ppx, hematologic malignancy & 21 & $0.5 \mathrm{mg} / \mathrm{L}$ & HPLC & {$[44]$} \\
\hline & IFI Ppx, hematologic malignancy & 31 & $0.5 \mathrm{mg} / \mathrm{L}$ & HPLC & {$[45]$} \\
\hline & IFI Ppx, cardiothoracic transplant & 11 & $0.5 \mathrm{mg} / \mathrm{L}$ & HPLC & {$[46]$} \\
\hline & IFI Ppx, hematologic malignancy & 467 & $0.7 \mathrm{mg} / \mathrm{L}$ & HPLC & {$[47]$} \\
\hline & IFI Ppx, HSCT with GVHD & 29 & $1.3 \mathrm{mg} / \mathrm{L}$ & HPLC & [48] \\
\hline & IFI Ppx, hematologic malignancy & 27 & No correlation & HPLC & [49] \\
\hline \multirow[t]{3}{*}{ Tx } & Invasive aspergillosis & 67 & $1.25 \mathrm{mg} / \mathrm{L}$ & HPLC & {$[50]$} \\
\hline & Chronic pulmonary aspergillosis & 66 & No correlation & HPLC & {$[51]$} \\
\hline & Disseminated or pulmonary coccidioidomycosis & 20 & No correlation & HPLC & {$[52]$} \\
\hline
\end{tabular}

Flucytosine - no studies exist linking clinical efficacy outcome data with target serum concentrations Ppx prophylaxis, Tx treatment, IFI invasive fungal infection, HSCT hematopoietic stem cell transplant, GVHD graft versus host disease include switching from the capsule formulation to the liquid, ensuring the liquid is taken on an empty stomach and the capsule with food, and elimination or reduction of interacting medications.

Recommendations for addressing supra-therapeutic itraconazole concentrations are even more limited. It is important to keep in mind that dose reduction should typically be reserved for patients who are actually experiencing adverse events and for whom alternate agents would be inappropriate.

\section{Voriconazole}

\section{Inter-Patient Variability}

Unlike itraconazole, voriconazole has dependable oral bioavailability [20]. However, there is still a high level of both inter- and intra-patient variability in dose-concentration relationship [41, 58]. This is attributed primarily to genetic polymorphisms in CYP2C19, which vary in prevalence based on ethnicity and can 
Table 2 Clinical guideline antifungal TDM recommendations

\begin{tabular}{|c|c|c|c|c|c|}
\hline \multirow[t]{2}{*}{ Antifungal } & \multirow[t]{2}{*}{ Source, year } & \multirow[t]{2}{*}{ When to draw first concentration } & \multicolumn{2}{|c|}{$\begin{array}{l}\text { Minimum trough concentration, } \\
\text { efficacy }(\mathrm{mg} / \mathrm{L})\end{array}$} & \multirow{2}{*}{$\begin{array}{l}\text { Maximum trough } \\
\text { concentration, } \\
\text { safety } \\
(\mathrm{mg} / \mathrm{L})^{\mathrm{a}}\end{array}$} \\
\hline & & & Prophylaxis & Treatment & \\
\hline \multirow[t]{4}{*}{ Itraconazole } & BSMM, 2014 [1] & Day 7 & 0.5 & 0.5 & 17 (bioassay) \\
\hline & $\begin{array}{l}\text { Andes et al. antifungal TDM, } \\
2009 \text { [2] }\end{array}$ & Day 4-7 & 0.5 & $1-2$ & None \\
\hline & $\begin{array}{l}\text { IDSA Blastomycosis guidelines, } \\
2008 \text { [53] }\end{array}$ & Day 14 & & 1 & 10 \\
\hline & $\begin{array}{l}\text { IDSA Histoplasmosis } \\
\text { guidelines, } \\
2007 \text { [54] }\end{array}$ & Day 14 & & 1 & 10 \\
\hline \multirow[t]{4}{*}{ Voriconazole } & BSMM, $2014[1]$ & $\begin{array}{l}\text { Day } 2-5 \text { (repeat to ensure therapeutic } \\
\text { at steady state if drawn before day } 5 \text { ) }\end{array}$ & 1 & 1 or trough:MIC of $2-5$ & $4-6$ \\
\hline & $\begin{array}{l}\text { Andes et al. antifungal TDM, } \\
2009 \text { [2] }\end{array}$ & Day 4-7 & 0.5 & $1-2$ & 6 \\
\hline & JSC/JSTDM, 2013 [6] & Day 5-7 & NR & $1-2$ & $4-5$ \\
\hline & $\begin{array}{l}\text { IDSA Histoplasmosis } \\
\text { guidelines, } \\
2007 \text { [54] }\end{array}$ & NR & & 0.5 & NR \\
\hline \multirow[t]{3}{*}{ Posaconazole } & BSMM, $2014[1]$ & Day 7 & 0.7 & 1 & None \\
\hline & $\begin{array}{l}\text { Andes et al. antifungal TDM, } \\
2009 \text { [2] }\end{array}$ & Day 4-7 & 0.5 & $0.5-1.5$ & None \\
\hline & $\begin{array}{l}\text { IDSA Histoplasmosis } \\
\text { guidelines, } \\
2007 \text { [54] }\end{array}$ & NR & & 0.5 & NR \\
\hline \multirow[t]{3}{*}{ Flucytosine } & BSMM, $2014[1]$ & Within $72 \mathrm{~h}$ & & $20-40$ & Peak $<100$ \\
\hline & $\begin{array}{l}\text { Andes et al. antifungal TDM, } \\
2009 \text { [2] }\end{array}$ & Day 3-5 & & Peak $>20$ & Peak $<50$ \\
\hline & $\begin{array}{l}\text { IDSA Cryptococcus guidelines, } \\
2010 \text { [55] }\end{array}$ & Day 3-5 & & $2 \mathrm{~h}$ post-dose $>30$ & Peak $\leq 80$ \\
\hline
\end{tabular}

BSMM British Society for Medical Mycology guidelines on TDM of antifungal drugs, JSC/JSTDM Japanese Society of Chemotherapy and Japanese Society of Therapeutic Drug Monitoring consensus guidelines for TDM of voriconazole, $N R$ no recommendation

${ }^{a}$ Concentrations imply measurement by chromatographic assay unless otherwise noted

lead to dramatic differences in drug exposure [59,60]. No dosage adjustments are currently recommended based on genotype, as the influence of genotype on voriconazole serum concentration is confounded by other factors such as drug-drug interactions [60]. Drugs that induce or inhibit CYP2C19, such as omeprazole, can also affect voriconazole concentrations [7].

Despite its good oral bioavailability, IV to oral transition also affects serum concentrations due to differences in approved dosing regimens. The approved intravenous dose is $4 \mathrm{mg} / \mathrm{kg}$ twice daily, while the approved oral dose is $200 \mathrm{mg}$ twice daily. This can lead to large differences in dosing and subsequently exposure, particularly in overweight patients, which has prompted some expert recommendations for oral dosing to approximate the $4-\mathrm{mg} / \mathrm{kg}-\mathrm{IV}$ dosing rounded to the nearest available tablet strength [61]. More recent data also suggests that oral bioavailability of voriconazole may be lower in patients being treated for invasive fungal infection than in healthy patients [62].

\section{Concentration-Efficacy Relationship}

Several studies have been published that support a relationship between voriconazole concentration and efficacy, while only one study failed to demonstrate a correlation (Table 1). Specific concentrations associated with successful therapy in these studies ranged from 0.25 to $2.2 \mathrm{mg} / \mathrm{L}$, with most studies supporting a concentration target in the $1-2-\mathrm{mg} / \mathrm{L}$ range. A meta-analysis of 12 studies including patients with deepseated mycosis found that trough concentrations over $1 \mathrm{mg} / \mathrm{L}$ were significantly associated with success in eradicating the infection (OR 7.23, $95 \%$ CI 2.84-18.36, $p<0.0001$ ) [63].

Perhaps the strongest evidence supporting TDM for voriconazole is a recent randomized controlled trial in which patients receiving voriconazole for treatment of invasive fungal infection who had their doses adjusted based on TDM to a target trough concentration of $1-5.5 \mathrm{mg} / \mathrm{L}$ had a significantly higher rate of either complete or partial response than patients without TDM [33].

Clinical practice guideline recommendations on efficacy targets for voriconazole are reported in Table 2 . The target of $0.5 \mathrm{mg} / \mathrm{L}$ recommended by the IDSA guidelines on treatment of histoplasmosis may be outdated, and guidelines incorporating more recent data recommend a target of at least $1 \mathrm{mg} / \mathrm{L}$ for treatment of invasive fungal infections $[1,2,6]$. A higher trough target of $2 \mathrm{mg} / \mathrm{L}$ may be considered 
based on the clinical situation; however, attempts at increasing voriconazole exposure to this point in all patients may lead to unacceptable toxicity. One potential strategy noted in the BSMM guidelines is to utilize a trough:MIC ratio in place of a static trough target, which is supported by an in vivo pharmacokinetic model as well as a Monte Carlo simulation of nine clinical trials $[64,65]$. Recommendations for target trough concentrations for prophylactic use of voriconazole range from 0.5 to $1 \mathrm{mg} / \mathrm{L}$, although support for these targets is much more limited than for targets for treatment of established disease $[1,2,29,30]$.

\section{Concentration-Toxicity Relationship}

Toxicities attributable to voriconazole include visual disturbances, hepatotoxicity, encephalopathy, and cutaneous phototoxicity [20]. Risk of visual disturbance, hepatotoxicity, and encephalopathy, all appear to correlate with increasing voriconazole concentration [35, 63, 66, 67]. While visual disturbances are dose dependent, they are also typically transient and self-limiting, and thus do not create a need for TDM in order to limit risk [2]. Neurologic toxicity has been associated with concentrations greater than 4-5.5 mg/L [35, 63]. Hepatotoxicity is more challenging, because while it does appear to be dose dependent, there is no clear concentration cut-off at which patients experience a significantly increased risk [63].

Published guidelines on TDM recommend that concentrations above 4-6 mg/L should be avoided (Table 2). Some experts argue that regular TDM, in order to avoid hepatotoxicity, is unnecessary because routine monitoring of LFTs should be sufficient $[6,68]$. However, utilization of TDM still has potential utility in establishing the most probable cause of hepatotoxicity in patients who have multiple risk factors, as well as in avoiding other adverse effects, such as encephalopathy, that would not otherwise be identified by routine laboratory monitoring of these patients.

\section{Recommendations}

Similarly, to itraconazole, clinical practice guidelines recommend consideration of TDM of voriconazole in the majority of patients $[1,2,6]$. Situations in which TDM is particularly attractive include populations with unpredictable pharmacokinetics, such as pediatric patients, patients receiving strongly interacting medications, or patients displaying signs of toxicity. Due to the disparity between intravenous and oral dosing regimens, the intravenous to oral transition is another point when TDM should be considered, particularly in overweight patients. Because much of the concentration variability with voriconazole is due to genetic polymorphisms in CYP2C19 and not due to absorption issues, even patients receiving IV therapy may have variable concentrations, and thus TDM can still be considered. However, if a patient is failing to respond to appropriate IV dosing of voriconazole for an invasive fungal infection, it may be preferable to switch to an alternate therapy as opposed to waiting for results of concentration monitoring.

While voriconazole reaches steady state earlier than itraconazole, Michaelis-Menten kinetics lead to variable half-life, so it is still important to wait an appropriate period of time prior to monitoring concentrations [20]. Both the JSC/ JSTDM guidelines and Andes et al. recommend waiting a minimum of 4-5 days prior to obtaining a concentration, while the BSMM guidelines state that a concentration can be obtained earlier than this as long as a second concentration is repeated a few days later to ensure that the drug is at steady state (Table 2). It is important to note that an apparent autoinduction of voriconazole metabolism can be seen in some patients, which has the potential to lead to large fluctuations in concentrations in patients who were initially therapeutic $[58,62]$. Therefore, concentrations should be followed over time in patients with invasive fungal diseases being managed with voriconazole. Similar to other azole antifungals, monitoring should be via trough concentrations.

\section{Dose Adjustments}

All patients with subtherapeutic voriconazole concentrations should be assessed for adherence, as well as interacting medications that can induce metabolism of voriconazole, such as rifampin. Dose adjustment algorithms have been published for voriconazole, which, along with the BSMM guidelines, recommend doubling or halving the dose of voriconazole for subor supra-therapeutic concentrations, respectively [1, 33, 35]. This was also the dose adjustment strategy used by Park et al. in their randomized controlled trial of TDM versus no TDM [33]. However, less aggressive dose titrations than this may be appropriate, particularly for patients close to target, since the Michaelis-Menten kinetics mean that small-dose adjustments can lead to dramatic changes in drug exposure, and smaller titrations are possible due to the availability of 50-mg tablets [69].

The BSMM guidelines recommend avoiding doses over $6 \mathrm{mg} / \mathrm{kg}$ twice daily for the IV formulation or $300 \mathrm{mg}$ twice daily for the oral formulation [1]. Per pharmacokinetic modeling however, many patients may need a higher oral dose than $300 \mathrm{mg}$ twice daily to achieve therapeutic concentrations $[62,69]$.

\section{Posaconazole}

\section{Inter-Patient Variability}

Similar to itraconazole and voriconazole, posaconazole exhibits significant inter-patient variability in dose-exposure 
relationships [70, 71]. This is predominantly due to inconsistent absorption of the oral suspension formulation. Posaconazole is a lipophilic molecule with very poor aqueous solubility, which requires administration with a high-fat meal or, less optimally, an acidic beverage [20, 42, 72]. Poor oral absorption has led to unacceptably high rates of subtherapeutic concentrations, with data published from a reference lab demonstrating that $16.3 \%$ of samples analyzed had undetectable concentrations, and $70.3 \%$ had concentrations below $0.7 \mathrm{mg} / \mathrm{L}$ [73].

A relatively new development that could dramatically alter TDM for posaconazole is the approval of delayed release posaconazole tablets, as well as an intravenous formulation. The tablet formulation achieved average steady state concentrations of $1.46 \mathrm{mg} / \mathrm{L}$ in a phase I trial of patients with acute myelogenous leukemia (AML) or myelodysplastic syndrome (MDS), which is substantially higher than the average posaconazole concentration of $0.58 \mathrm{mg} / \mathrm{L}$ seen in the same patient population receiving the suspension [74]. The tablet is also less affected by food or stomach $\mathrm{pH}$, although it is still recommended to be administered with a meal [75]. The intravenous formulation also achieved average serum posaconazole concentrations greater than $1 \mathrm{mg} / \mathrm{L}$ [76]. These more dependable exposure results reduce the need for TDM with the newer formulations.

\section{Concentration-Efficacy Relationship}

Several small observational studies have supported an exposure-efficacy relationship for posaconazole when used for prophylaxis of invasive fungal infections in immunocompromised patients, most of which support a target above 0.5$0.7 \mathrm{mg} / \mathrm{L}$ (Table 1). The most convincing evidence comes from an FDA briefing document compiled from the results of two large observational studies, which found that 12 out of 15 breakthrough fungal infections occurred in patients with serum posaconazole concentrations below $0.7 \mathrm{mg} / \mathrm{L}$ [47]. These results are reflected in the target concentrations recommended by published guidelines for posaconazole prophylaxis (Table 2).

Data are much more limited on the exposure-response relationship for posaconazole when used to treat established invasive fungal infections. Walsh et al. found a positive correlation between exposure and response in patients receiving salvage treatment for invasive aspergillosis, with the highest response rate in patients with a mean average concentration of $1.25 \mathrm{mg} / \mathrm{L}$ [50]. Other published studies did not reflect a relationship between initial posaconazole concentrations and response in the treatment of invasive fungal infections; however, mean posaconazole concentrations in both studies were greater than $1.25 \mathrm{mg} / \mathrm{L}$, which may have limited the ability to detect a relationship [51, 52]. As can be expected from the small amount of data available, guideline recommendations vary widely in the recommended target posaconazole concentration for these patients (Table 2), but a trough concentration of $1 \mathrm{mg} / \mathrm{L}$ appears to be a reasonable minimum goal.

\section{Concentration-Toxicity Relationship}

Unlike other antifungals, a relationship between concentration and toxicity has not been documented for posaconazole. In the previously referenced FDA briefing document, adverse effects did not vary significantly based on posaconazole concentration [47]. Thus, at this point, no upper limit is recommended in order to limit toxicity (Table 2). Questions remain about whether the higher posaconazole exposures achieved with the intravenous and delayed release tablet formulations will lead to new dose-dependent toxicities. While a much larger proportion of patients receiving these formulations experience concentrations over $2.5 \mathrm{mg} / \mathrm{L}$, thus far there have been no differences seen in adverse events between these formulations and the oral suspension [76].

\section{Recommendations}

The BSMM guidelines recommend that TDM be conducted for the majority of patients receiving posaconazole at the initiation of therapy [1]. Based on the known exposure-response relationship and the substantial issues with oral absorption of posaconazole suspension, TDM of patients receiving this formulation is recommended. This is especially true of patients who cannot comply with the dietary recommendations or are receiving acid suppressants.

There is no apparent need to perform TDM for the intravenous formulation of posaconazole as inter-patient variability is due to variable oral absorption, and $100 \%$ bioavailability is ensured with intravenous administration. Necessity of TDM with the delayed release tablet formulation is less clear at this point. In the phase I study in AML or MDS patients, the $300 \mathrm{mg}$ once daily dose of the tablet achieved average serum posaconazole concentrations over $0.5 \mathrm{mg} / \mathrm{L}$ in $100 \%$ of patients [77]. It therefore seems that routine TDM would be unnecessary for patients receiving prophylaxis with the tablet formulation. It is possible that there may still be a need for TDM in patients requiring higher exposures for treatment of invasive fungal infections, but this can likely be limited to situations where there is significant concern for altered oral absorption, such as severe mucositis or prolonged severe diarrhea.

Posaconazole suspension does not reach steady state until 1 week after initiation or dose changes [5]. Therefore, it is recommended to wait until day 7 of therapy prior to obtaining a concentration [1]. One published dosing algorithm uses a concentration of at least $0.35 \mathrm{mg} / \mathrm{L} 48 \mathrm{~h}$ after initiation as a surrogate for therapeutic steady state concentrations and recommends dose adjustments at that point. [47] Since its 
pharmacokinetics are relatively predictable, this is a more reasonable strategy for posaconazole relative to the other azoles. Similar to other azole antifungals, it is recommended that concentrations be drawn as troughs; however, most studies documenting concentration-response relationship utilize average concentrations. Similarly, to itraconazole, posaconazole's long half-life means that random and trough concentrations should not differ significantly [20].

\section{Dose Adjustments}

A recently published dosing algorithm for posaconazole demonstrated that patients with subtherapeutic concentrations on standard prophylaxis dosing of $200 \mathrm{mg}$ three times daily can reach target concentrations after a dose increase to either $300 \mathrm{mg}$ three times daily or $200 \mathrm{mg}$ four times daily [78]. However, due to saturable oral absorption, dose increases past this point are unlikely to result in further increases in serum concentration [46]. Because there has not been a demonstrated upper limit for posaconazole concentrations to prevent toxicity, dose reduction based on TDM is not warranted.

Due to its saturable oral absorption, it is important to consider strategies outside of dose titration to increase posaconazole exposure, including ensuring the patient is receiving the suspension with a fatty meal or acidic beverage, eliminating interacting medications such as acid suppressants, and dividing the daily dose so that it is administered four times daily. Smaller, more frequent administrations (i.e., $200 \mathrm{mg}$ four times daily) achieve higher serum concentrations than larger, less frequent administrations (i.e., $400 \mathrm{mg}$ twice daily). Patients who cannot achieve desired concentrations on posaconazole suspension despite optimal administration can be transitioned to the delayed release tablet formulation-this was demonstrated to dramatically increase exposure in one case series [79].

\section{Isavuconazole}

A new azole antifungal, isavuconazole, has recently been approved for use based on $r$ phase III trials in which it demonstrated non-inferiority to voriconazole in the treatment of invasive aspergillosis with significantly fewer adverse effects [80]. Isavuconazole has a number of attractive features, including both intravenous and oral formulations, a long half-life that allows for once daily dosing, excellent oral bioavailability, few adverse effects, and a wide spectrum of activity including Candida, Aspergillus, and some Mucorales [81-84].

Many of the factors contributing to variability in serum concentrations of other azole antifungals are not present with isavuconazole, as it demonstrates excellent oral bioavailability that is unaffected by food as well as linear pharmacokinetics $[85,86]$. In line with this information, Schmitt-Hoffmann et al. demonstrated that there was only low to moderate intersubject variability in serum concentrations [87]. Thus, while any definite conclusions must be deferred until there is more clinical experience with isavuconazole, early evidence suggests that the apparent need for TDM of the drug is low.

\section{Flucytosine}

\section{Inter-Patient Variability}

Flucytosine is almost exclusively eliminated as unchanged drug via the kidneys [17]. Thus, changes in renal function can have a dramatic effect on flucytosine serum concentrations. Given that current use of the drug is nearly always in combination with the highly nephrotoxic amphotericin B for treatment of cryptococcal meningitis, changes in renal function and therefore changes in flucytosine exposure can be expected [88].

High levels of variability in serum concentrations of flucytosine have been documented, with retrospective reviews of concentrations measured in both adult and pediatric populations demonstrating that only about $20 \%$ of serum concentrations analyzed were within therapeutic range [88, 89]. However, there are significant limitations to these data given that flucytosine doses were not recorded. In addition, definitions for therapeutic serum concentrations used were largely arbitrary due to an overall lack of clinical data supporting concentration targets.

\section{Concentration-Efficacy Relationship}

There is a paucity of data supporting specific concentration targets for efficacy of flucytosine, with the limited data available coming from murine models of invasive candidiasis [90, 91]. These models demonstrate that time $>$ MIC is the parameter most associated with success of flucytosine therapy, with the optimal target being $25 \%$ of the dosing interval in one study [91] and $45 \%$ in another [90]. It is unknown if these targets can be applied to cryptococcal infections, and there is currently no clinical efficacy data in humans supporting a specific flucytosine concentration target.

One potential rationale for minimum flucytosine concentration targets in the absence of strong correlations with efficacy is the prevention of resistance. In an in vitro study, concentrations below $25 \mathrm{mg} / \mathrm{L}$ were associated with development of resistant mutants in Candida albicans and Candida glabrata [92]. This is the evidence cited by major clinical practice guidelines to justify current minimum concentration targets (Table 2). It is important to note that this study was examining flucytosine alone, and it is possible that lower 
concentration targets are acceptable when the drug is used in combination in accordance with its current role in therapy [88].

\section{Concentration-Toxicity Relationship}

TDM for flucytosine has routinely been considered the standard of care primarily for the reason of toxicity prevention. Elevated flucytosine concentrations have been linked to both myelosuppression and hepatotoxicity [93-95]. In the largest study examining flucytosine toxicity, $62 \%$ of patients with prolonged flucytosine peak concentrations of over $100 \mathrm{mg} / \mathrm{L}$ experienced flucytosine toxicity versus $31 \%$ without concentrations over $100 \mathrm{mg} / \mathrm{L}$ [93]. Of note, the authors of this study defined peak concentrations as concentrations drawn $2 \mathrm{~h}$ after a dose as opposed to the more traditional definition of $30 \mathrm{~min}$ after a dose. Also, they used a dose of $150 \mathrm{mg} / \mathrm{kg} /$ day in patients with normal renal function, which is higher than the currently recommended dose of $100 \mathrm{mg} / \mathrm{kg} /$ day for cryptococcal meningitis [88]. In a small study, out of 16 patients that received oral flucytosine dosed at $100 \mathrm{mg} / \mathrm{kg} / \mathrm{day}$, none had peak concentrations over $75 \mathrm{mg} / \mathrm{L}$ [96].

Clinical practice guideline recommendations for minimum flucytosine concentrations to prevent toxicity are presented in Table 2. The cut-offs recommended vary significantly and, given that the only concentrations definitively linked to toxicity are those exceeding $100 \mathrm{mg} / \mathrm{L}$, exact cut-offs below this point are somewhat arbitrary. However, there is evidence that lower doses than those currently used may provide equivalent efficacy in the treatment of cryptococcal meningitis [97]. Given that flucytosine demonstrates time-dependent killing and has very weak concentration-dependent effects, it is reasonable to target peak concentrations well below the traditional cut-off of $100 \mathrm{mg} / \mathrm{L}$ so long as adequate time $>\mathrm{MIC}$ is achieved.

\section{Recommendations}

All major published guidelines commenting on TDM of flucytosine recommend that concentrations be monitored early in the course of therapy in the majority of patients to minimize the risk of toxicity (Table 2). Because myelosuppression is a delayed, progressive toxicity, checking a peak concentration early in the course of therapy is an important step to preventing this from occurring [95]. In patients who have acceptable concentrations, it is not necessary to continue to check concentrations so long as renal function is monitored closely, and the flucytosine dose is appropriately renally adjusted.

In patients receiving flucytosine orally at recommended doses, peak concentrations and trough concentrations are not significantly different [95]. Thus, it is reasonable to only monitor peak concentrations.

\section{Dose Adjustments}

Recommendations regarding how to adjust flucytosine dose based on sub- or supra-therapeutic concentrations are sparse. The BSMM guidelines recommend that the dose should be increased by $50 \%$ in patients with a subtherapeutic concentration [1]. Given the extremely limited data supporting minimum flucytosine concentrations and the definite risk associated with supra-therapeutic concentrations, dose increases should be implemented with caution. No recommendations are available on how to address a supra-therapeutic peak concentration, but given the linear pharmacokinetics and the minimal concentration-dependent killing of the drug, doses can be lowered fairly aggressively and proportionally in patients with concentrations over $100 \mathrm{mg} / \mathrm{L}$.

\section{Conclusion}

There is a growing body of literature that supports the utility of TDM for flucytosine, itraconazole, voriconazole, and posaconazole suspension. All of these agents demonstrate the variability in drug exposure and clear correlations between exposure and efficacy, toxicity, or both that make them ideal candidates for TDM. The role of TDM in newer agents such as posaconazole delayed release tablets and isavuconazole is still unclear, but preliminary data suggest that it may not be needed. Additionally, clinicians must remember that current concentration target recommendations are based on observational studies with varying patient populations and significant limitations. Thus, the decision on whether or not to conduct TDM as well as the specific concentration to target must be assessed on a patient-by-patient basis.

\section{Compliance with Ethics Guidelines}

Conflict of Interest The authors state that there are no conflicts of interest to declare.

Human and Animal Rights and Informed Consent This article contains no studies with human or animal subjects.

\section{References}

Papers of particular interest, published recently, have been highlighted as:

- Of importance

•- Of major importance

1.• Ashbee HR et al. Therapeutic drug monitoring (TDM) of antifungal agents: guidelines from the British Society for Medical Mycology. J Antimicrob Chemother. 2014;69(5):1162-76. Comprehensive clinical guidelines from the British Society for Medical 
Mycology on the use of TDM for itraconazole, voriconazole, posaconazole, and flucytosine. The authors summarize relevant literature and provide recommendations for TDM.

2. Andes D, Pascual A, Marchetti O. Antifungal therapeutic drug monitoring: established and emerging indications. Antimicrob Agents Chemother. 2009;53(1):24-34.

3. Hope WW et al. Therapeutic drug monitoring for triazoles. Curr Opin Infect Dis. 2008;21(6):580-6.

4. Smith J, Andes D. Therapeutic drug monitoring of antifungals: pharmacokinetic and pharmacodynamic considerations. Ther Drug Monit. 2008;30(2):167-72.

5. Howard SJ et al. Posaconazole: the case for therapeutic drug monitoring. Ther Drug Monit. 2012;34(1):72-6.

$6 . \bullet$ Hamada Y et al. Practice guidelines for therapeutic drug monitoring of voriconazole: a consensus review of the Japanese Society of Chemotherapy and the Japanese Society of Therapeutic Drug Monitoring. J Infect Chemother. 2013;19(3):381-92. Clinical guidelines on TDM for voriconazole from the Japanese Society of Chemotherapy and the Japanese Society of Therapeutic Drug Monitoring. Provides an excellent summary of the literature supporting TDM of voriconazole. This guideline does not address other antifungal agents for which TDM is conducted.

7. Bruggemann RJ et al. Clinical relevance of the pharmacokinetic interactions of azole antifungal drugs with other coadministered agents. Clin Infect Dis. 2009;48(10):1441-58.

8. Kim $\mathrm{H}$ et al. Use of high-performance liquid chromatographic and microbiological analyses for evaluating the presence or absence of active metabolites of the antifungal posaconazole in human plasma. J Chromatogr A. 2003;987(1-2):243-8.

9. Law D, Moore CB, Denning DW. Bioassay for serum itraconazole concentrations using hydroxyitraconazole standards. Antimicrob Agents Chemother. 1994;38(7):1561-6.

10. Muller $\mathrm{C}$ et al. HPLC analysis of the antifungal agent posaconazole in patients with haematological diseases. Mycoses. 2006;49 Suppl $1: 17-22$.

11. Chhun $\mathrm{S}$ et al. Simultaneous quantification of voriconazole and posaconazole in human plasma by high-performance liquid chromatography with ultra-violet detection. J Chromatogr B Analyt Technol Biomed Life Sci. 2007;852(1-2):223-8.

12. Hong $\mathrm{Y}$ et al. Population pharmacokinetics of liposomal amphotericin B in pediatric patients with malignant diseases. Antimicrob Agents Chemother. 2006;50(3):935-42.

13. Scynexis, I. Open-label, randomized study to estimate safety, PK, and efficacy of oral SCY-078 vs. standard-of-care following IV micafungin therapy in the treatment of invasive candidiasis in hospitalized nonneutropenic adults. 2014 [cited 2014 October 27]; Available from: http://clinicaltrials.gov/show/NCT02244606.

14. Clancy CJ et al. Fluconazole MIC and the fluconazole dose/MIC ratio correlate with therapeutic response among patients with candidemia. Antimicrob Agents Chemother. 2005;49(8):3171-7.

15. Pai MP, Turpin RS, Garey KW. Association of fluconazole area under the concentration-time curve/MIC and dose/MIC ratios with mortality in nonneutropenic patients with candidemia. Antimicrob Agents Chemother. 2007;51(1):35-9.

16. Pfaller MA, Diekema DJ, Sheehan DJ. Interpretive breakpoints for fluconazole and Candida revisited: a blueprint for the future of antifungal susceptibility testing. Clin Microbiol Rev. 2006;19(2): 435-47.

17. Dodds-Ashley E et al. Pharmacology of systemic antifungal agents. Clin Infect Dis. 2006;43:S28-39.

18. van der Elst $\mathrm{KC}$, et al. Insufficient fluconazole exposure in pediatric cancer patients and the need for therapeutic drug monitoring in critically Ill children. Clin Infect Dis. 2014

19. Cartledge JD, Midgely J, Gazzard BG. Itraconazole solution: higher serum drug concentrations and better clinical response rates than the capsule formulation in acquired immunodeficiency syndrome patients with candidiasis. J Clin Pathol. 1997;50(6):477-80.

20. Lewis RE. Current concepts in antifungal pharmacology. Mayo Clin Proc. 2011;86(8):805-17.

21. Boogaerts MA et al. Antifungal prophylaxis with itraconazole in prolonged neutropenia: correlation with plasma levels. Mycoses. 1989;32 Suppl 1:103-8.

22. Tricot $\mathrm{G}$ et al. Ketoconazole vs. itraconazole for antifungal prophylaxis in patients with severe granulocytopenia: preliminary results of two nonrandomized studies. Rev Infect Dis. 1987;9 Suppl 1:S94-9.

23. Glasmacher A et al. Breakthrough invasive fungal infections in neutropenic patients after prophylaxis with itraconazole. Mycoses. 1999;42(7-8):443-51.

24. Sharkey PK et al. High-dose itraconazole in the treatment of severe mycoses. Antimicrob Agents Chemother. 1991;35(4):707-13.

25. Denning DW et al. Treatment of invasive aspergillosis with itraconazole. Am J Med. 1989;86(6 Pt 2):791-800.

26. Tucker RM et al. Itraconazole therapy for nonmeningeal coccidioidomycosis: clinical and laboratory observations. J Am Acad Dermatol. 1990;23(3 Pt 2):593-601.

27. Cross LJ et al. Serum itraconazole concentrations and clinical responses in Candida-associated denture stomatitis patients treated with itraconazole solution and itraconazole capsules. J Antimicrob Chemother. 2000;45(1):95-9.

28. Denning DW et al. Itraconazole therapy for cryptococcal meningitis and cryptococcosis. Arch Intern Med. 1989;149(10):2301-8.

29. Mitsani D et al. Prospective, observational study of voriconazole therapeutic drug monitoring among lung transplant recipients receiving prophylaxis: factors impacting levels of and associations between serum troughs, efficacy, and toxicity. Antimicrob Agents Chemother. 2012;56(5):2371-7.

30. Trifilio $\mathrm{S}$ et al. Breakthrough fungal infections after allogeneic hematopoietic stem cell transplantation in patients on prophylactic voriconazole. Bone Marrow Transplant. 2007;40(5):451-6.

31. Denning DW et al. Efficacy and safety of voriconazole in the treatment of acute invasive aspergillosis. Clin Infect Dis. 2002;34(5): 563-71.

32. Gomez-Lopez A et al. Voriconazole serum levels measured by high-performance liquid chromatography: a monocentric study in treated patients. Med Mycol. 2012;50(4):439-45.

33.• Park WB et al. The effect of therapeutic drug monitoring on safety and efficacy of voriconazole in invasive fungal infections: a randomized controlled trial. Clin Infect Dis. 2012;55(8):1080-7. The first, and currently only, randomized controlled trial specifically assessing the effect of TDM of voriconazole on patient outcomes. Patients who had TDM performed and doses adjusted to a target trough concentration of $1-5.5 \mathrm{mg} / \mathrm{L}$ had significantly better rates of complete or partial response vs. those who did not have TDM performed $(81 \%$ vs. $59 \%, p=0.04)$.

34. Soler-Palacin P et al. Voriconazole drug monitoring in the management of invasive fungal infection in immunocompromised children: a prospective study. J Antimicrob Chemother. 2012;67(3):700-6.

35. Pascual A et al. Voriconazole therapeutic drug monitoring in patients with invasive mycoses improves efficacy and safety outcomes. Clin Infect Dis. 2008;46(2):201-11.

36. Dolton MJ et al. Multicenter study of voriconazole pharmacokinetics and therapeutic drug monitoring. Antimicrob Agents Chemother. 2012;56(9):4793-9.

37. Ueda $\mathrm{K}$ et al. Monitoring trough concentration of voriconazole is important to ensure successful antifungal therapy and to avoid hepatic damage in patients with hematological disorders. Int $\mathbf{J}$ Hematol. 2009;89(5):592-9.

38. Okuda $\mathrm{T}$ et al. Retrospective serological tests for determining the optimal blood concentration of voriconazole for treating fungal infection. Yakugaku Zasshi. 2008;128(12):1811-8. 
39. Smith $\mathrm{J}$ et al. Voriconazole therapeutic drug monitoring. Antimicrob Agents Chemother. 2006;50(4):1570-2.

40. Miyakis $\mathrm{S}$ et al. Voriconazole concentrations and outcome of invasive fungal infections. Clin Microbiol Infect. 2010;16(7):927-33.

41. Racil $\mathrm{Z}$ et al. Monitoring trough voriconazole plasma concentrations in haematological patients: real life multicentre experience. Mycoses. 2012;55(6):483-92.

42. Eiden $\mathrm{C}$ et al. Therapeutic drug monitoring of posaconazole in hematology adults under posaconazole prophylaxis: influence of food intake. Eur J Clin Microbiol Infect Dis. 2012;31(2):161-7.

43. Lebeaux $\mathrm{D}$ et al. Therapeutic drug monitoring of posaconazole: a monocentric study with 54 adults. Antimicrob Agents Chemother. 2009;53(12):5224-9.

44. Bryant $\mathrm{AM}$ et al. A post-marketing evaluation of posaconazole plasma concentrations in neutropenic patients with haematological malignancy receiving posaconazole prophylaxis. Int J Antimicrob Agents. 2011;37(3):266-9.

45. Hoenigl $\mathrm{M}$ et al. Posaconazole plasma concentrations and invasive mould infections in patients with haematological malignancies. Int $\mathbf{J}$ Antimicrob Agents. 2012;39(6):510-3

46. Shields RK et al. Posaconazole serum concentrations among cardiothoracic transplant recipients: factors impacting trough levels and correlation with clinical response to therapy. Antimicrob Agents Chemother. 2011;55(3):1308-11.

47. Jang SH, Colangelo PM, Gobburu JV. Exposure-response of posaconazole used for prophylaxis against invasive fungal infections: evaluating the need to adjust doses based on drug concentrations in plasma. Clin Pharmacol Ther. 2010;88(1):115-9.

48. Tonini $\mathrm{J}$ et al. Therapeutic drug monitoring of posaconazole in allogeneic hematopoietic stem cell transplantation patients who develop gastrointestinal graft-versus-host disease. Antimicrob Agents Chemother. 2012;56(10):5247-52.

49. Neubauer WC et al. Therapeutic drug monitoring of posaconazole in hematology patients: experience with a new high-performance liquid chromatography-based method. Antimicrob Agents Chemother. 2010;54(9):4029-32.

50. Walsh TJ et al. Treatment of invasive aspergillosis with posaconazole in patients who are refractory to or intolerant of conventional therapy: an externally controlled trial. Clin Infect Dis. 2007;44(1):2-12.

51. Felton TW et al. Efficacy and safety of posaconazole for chronic pulmonary aspergillosis. Clin Infect Dis. 2010;51(12):1383-91.

52. Catanzaro A et al. Safety, tolerance, and efficacy of posaconazole therapy in patients with nonmeningeal disseminated or chronic pulmonary coccidioidomycosis. Clin Infect Dis. 2007;45(5):562-8.

53. Chapman SW et al. Clinical practice guidelines for the management of blastomycosis: 2008 update by the Infectious Diseases Society of America. Clin Infect Dis. 2008;46(12):1801-12.

54. Wheat LJ et al. Clinical practice guidelines for the management of patients with histoplasmosis: 2007 update by the Infectious Diseases Society of America. Clin Infect Dis. 2007;45(7):807-25.

55. Perfect JR, Dismukes W, Dromer F, Goldman DL. Clinical practice guidelines for the management of cryptococcal disease: 2010 update by the Infectious Diseases Society of America. Clin Infect Dis. 2010;50(3):291-322.

56. Lionakis MS, Samonis G, Kontoyiannis DP. Endocrine and metabolic manifestations of invasive fungal infections and systemic antifungal treatment. Mayo Clin Proc. 2008;83(9):1046-60.

57. Lestner JM et al. Toxicodynamics of itraconazole: implications for therapeutic drug monitoring. Clin Infect Dis. 2009;49(6):928-30.

58. Trifilio SM et al. Serial plasma voriconazole concentrations after allogeneic hematopoietic stem cell transplantation. Antimicrob Agents Chemother. 2009;53(5):1793-6.

59. Ikeda $\mathrm{Y}$ et al. Pharmacokinetics of voriconazole and cytochrome P450 2C19 genetic status. Clin Pharmacol Ther. 2004;75(6):587-8.
60. Hyland R, Jones BC, Smith DA. Identification of the cytochrome P450 enzymes involved in the N-oxidation of voriconazole. Drug Metab Dispos. 2003;31(5):540-7.

61. Walsh TJ et al. Treatment of aspergillosis: clinical practice guidelines of the Infectious Diseases Society of America. Clin Infect Dis. 2008;46(3):327-60.

62. Pascual A et al. Challenging recommended oral and intravenous voriconazole doses for improved efficacy and safety: population pharmacokinetics-based analysis of adult patients with invasive fungal infections. Clin Infect Dis. 2012;55(3):381-90.

63. Hamada $\mathrm{Y}$ et al. Investigation and threshold of optimum blood concentration of voriconazole: a descriptive statistical meta-analysis. J Infect Chemother. 2012;18(4):501-7.

64. Jeans AR et al. Combination of voriconazole and anidulafungin for treatment of triazole-resistant aspergillus fumigatus in an in vitro model of invasive pulmonary aspergillosis. Antimicrob Agents Chemother. 2012;56(10):5180-5.

65. Troke PF, Hockey HP, Hope WW. Observational study of the clinical efficacy of voriconazole and its relationship to plasma concentrations in patients. Antimicrob Agents Chemother. 2011;55(10): 4782-8.

66. Potoski BA, Brown J. The safety of voriconazole. Clin Infect Dis. 2002;35(10):1273-5.

67. Lutsar I et al. Safety of voriconazole and dose individualization. Clin Infect Dis. 2003;36(8):1087-8.

68. Tan $\mathrm{K}$ et al. Investigation of the potential relationships between plasma voriconazole concentrations and visual adverse events or liver function test abnormalities. J Clin Pharmacol. 2006;46(2): 235-43.

69. Dolton $\mathrm{MJ}$ et al. Understanding variability with voriconazole using a population pharmacokinetic approach: implications for optimal dosing. J Antimicrob Chemother. 2014;69(6):1633-41.

70. Noxafil. EPAR - scientific discussion, E.M. Agency, Editor. London, UK 2005.

71. Ezzet $\mathrm{F}$ et al. Oral bioavailability of posaconazole in fasted healthy subjects: comparison between three regimens and basis for clinical dosage recommendations. Clin Pharmacokinet. 2005;44(2): 211-20.

72. Krishna $\mathrm{G}$ et al. Pharmacokinetics and absorption of posaconazole oral suspension under various gastric conditions in healthy volunteers. Antimicrob Agents Chemother. 2009;53(3):958-66.

73. Thompson 3rd GR et al. Posaconazole therapeutic drug monitoring: a reference laboratory experience. Antimicrob Agents Chemother. 2009;53(5):2223-4.

74. Krishna $\mathrm{G}$ et al. Pharmacokinetics of oral posaconazole in neutropenic patients receiving chemotherapy for acute myelogenous leukemia or myelodysplastic syndrome. Pharmacotherapy. 2008;28(10):1223-32.

75. Kraft WK et al. Posaconazole tablet pharmacokinetics: lack of effect of concomitant medications altering gastric $\mathrm{pH}$ and gastric motility in healthy subjects. Antimicrob Agents Chemother. 2014;58(7):4020-5.

76. Merck \& Co, I., Noxafil package insert. Whitehouse Station, New Jersey 2014.

77. Duarte RF et al. Phase $1 \mathrm{~b}$ study of new posaconazole tablet for prevention of invasive fungal infections in high-risk patients with neutropenia. Antimicrob Agents Chemother. 2014;58(10): 5758-65.

78. Hummert SE, Green MR. Therapeutic drug monitoring and dose adjustment of posaconazole oral suspension in adults with acute myeloid leukemia. Ther Drug Monit. 2014.

79. Jung DS, Tverdek FP, Kontoyiannis DP. Switching from posaconazole suspension to tablets increases serum drug levels in leukemia patients without clinically relevant hepatotoxicity. Antimicrob Agents Chemother. 2014;58(11):6993-5. 
80. Kontoyiannis D, et al. A Phase 3, randomized, double-blind, noninferiority trial to evaluate efficacy and safety of isavuconazole versus voriconazole in patients with invasive mold disease (SECURE): outcomes in invasive aspergillosis patients. in ID Week. Philadelphia, PA 2014.

81. Falci DR, Pasqualotto AC. Profile of isavuconazole and its potential in the treatment of severe invasive fungal infections. Infect Drug Resist. 2013;6:163-74.

82. Schmitt-Hoffmann A et al. Single-ascending-dose pharmacokinetics and safety of the novel broad-spectrum antifungal triazole BAL4815 after intravenous infusions (50, 100, and 200 milligrams) and oral administrations (100, 200, and 400 milligrams) of its prodrug, BAL8557, in healthy volunteers. Antimicrob Agents Chemother. 2006;50(1):279-85.

83. Thompson 3rd GR, Wiederhold NP. Isavuconazole: a comprehensive review of spectrum of activity of a new triazole. Mycopathologia. 2010;170(5):291-313.

84. Verweij PE et al. In vitro antifungal activity of isavuconazole against 345 mucorales isolates collected at study centers in eight countries. J Chemother. 2009;21(3):272-81.

85. Warn PA et al. Pharmacokinetics and pharmacodynamics of a novel triazole, isavuconazole: mathematical modeling, importance of tissue concentrations, and impact of immune status on antifungal effect. Antimicrob Agents Chemother. 2009;53(8):3453-61.

86. Schmitt-Hoffmann A. et al. No relevant food effect in man on isavuconazole oral pharmacokinetics preliminary data., in 48th Annual International Conference on Antimicrobial Agents and Chemotherapy (ICAAC). Washington, DC 2008.

87. Schmitt-Hoffmann A et al. Multiple-dose pharmacokinetics and safety of the new antifungal triazole BAL4815 after intravenous infusion and oral administration of its prodrug, BAL8557, in healthy volunteers. Antimicrob Agents Chemother. 2006;50(1): 286-93.
88. Pasqualotto AC et al. Flucytosine therapeutic monitoring: 15 years experience from the UK. J Antimicrob Chemother. 2007;59(4): 791-3.

89. Soltani $\mathrm{M}$ et al. Evidence of excessive concentrations of 5flucytosine in children aged below 12 years: a 12-year review of serum concentrations from a UK clinical assay reference laboratory. Int J Antimicrob Agents. 2006;28(6):574-7.

90. Hope WW et al. Derivation of an in vivo drug exposure breakpoint for flucytosine against Candida albicans and impact of the MIC, growth rate, and resistance genotype on the antifungal effect. Antimicrob Agents Chemother. 2006;50(11):3680-8.

91. Andes D, van Ogtrop M. In vivo characterization of the pharmacodynamics of flucytosine in a neutropenic murine disseminated candidiasis model. Antimicrob Agents Chemother. 2000;44(4): 938-42.

92. Normark S, Schonebeck J. In vitro studies of 5-fluorocytosine resistance in Candida albicans and Torulopsis glabrata. Antimicrob Agents Chemother. 1972;2(3):114-21.

93. Stamm AM et al. Toxicity of amphotericin B plus flucytosine in 194 patients with cryptococcal meningitis. Am J Med. 1987;83(2): 236-42.

94. Kauffman CA, Frame PT. Bone marrow toxicity associated with 5fluorocytosine therapy. Antimicrob Agents Chemother. 1977;11(2): 244-7.

95. Francis P, Walsh TJ. Evolving role of flucytosine in immunocompromised patients: new insights into safety, pharmacokinetics, and antifungal therapy. Clin Infect Dis. 1992;15(6):1003-18.

96. Brouwer AE et al. Oral versus intravenous flucytosine in patients with human immunodeficiency virus-associated cryptococcal meningitis. Antimicrob Agents Chemother. 2007;51(3):1038-42.

97. O'Connor L et al. Pharmacodynamics of liposomal amphotericin B and flucytosine for cryptococcal meningoencephalitis: safe and effective regimens for immunocompromised patients. J Infect Dis. 2013;208(2):351-61. 